\title{
Investigation of Oxygen Permeation through Composites of PMMA and Surface-Modified ZnO Nanoparticles
}

\author{
Sandra Hess, Mustafa M. Demir, Vladimir Yakutkin, Stanislav Baluschev, \\ Gerhard Wegner*
}

Oxygen permeabilities of nanocomposite films consisting of poly(methyl methacrylate) (PMMA) and different amounts of spherical zinc oxide (ZnO) nanoparticles were determined to investigate the barrier effect of this material with respect to particle content. A method was applied which is based on quenching of an excited phosphorescent dye by oxygen. Possible effects of the nanoparticles on the response of the dye molecules were investigated and were ruled out.

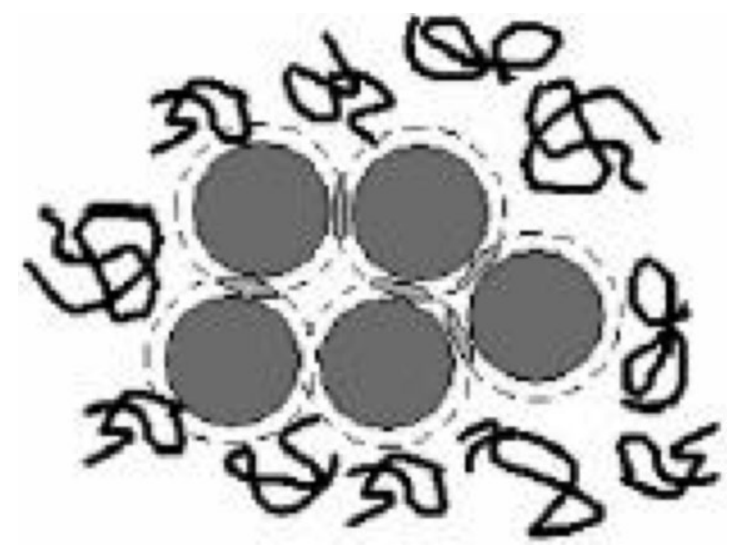

\section{Introduction}

Nanocomposites consisting of nanosized inorganic particles distributed in a polymer matrix are suggested as promising materials for different applications in areas like catalysis, organic batteries, electronics, and optoelectronics. ${ }^{[1]}$ The presence of nanoparticles in the polymer often changes the material properties of the matrix material significantly: enhanced electrical or thermal conductivity, improvements of thermal and mechanical properties (e.g., toughness, modulus, and scratch-resistance) as well as desired optical properties have been reported. ${ }^{[1,2]}$ Additionally, and compared to the pure polymer, incorporation

G. Wegner, V. Yakutkin, S. Baluschev

Max-Planck Institute for Polymer Research, Ackermannweg 10, D-55128 Mainz, Germany

Fax: +(49)-6131-379-330; E-mail: wegner@mpip-mainz.mpg.de S. Hess, M. M. Demir

Izmir Institute of Technology, Department of Chemistry,

Urla TR-35430 Izmir, Turkey of inorganic nanoparticles in a polymer matrix may affect the permeation of gases. Concerning such permeation properties of nanocomposites, two important applications are discussed in the literature: barrier materials as well as gas separation membranes. In the first case the nanoparticles are assumed to act as obstacles impeding the permeation of gas molecules through the film. ${ }^{[3-9]}$ On the other hand, nanocomposite gas separation membranes have been described in which the nanoparticles seemed to enhance the gas transport properties of the film relative to the corresponding pure matrix material. ${ }^{[2,10-15]}$ Such contradictory results show that a simple and generalizing explanation for the influence of nanoparticles on the permeability of composite materials cannot be given.

Maxwells model is often used to describe the permeability in films containing roughly spherical impermeable particles. ${ }^{[2,3,10]}$ It was originally developed to analyze dielectric properties of a diluted suspension of spheres. According to this model incorporation of particles in a polymer matrix leads to reduced gas permeability: The presence of nanosized materials in the polymer reduces 


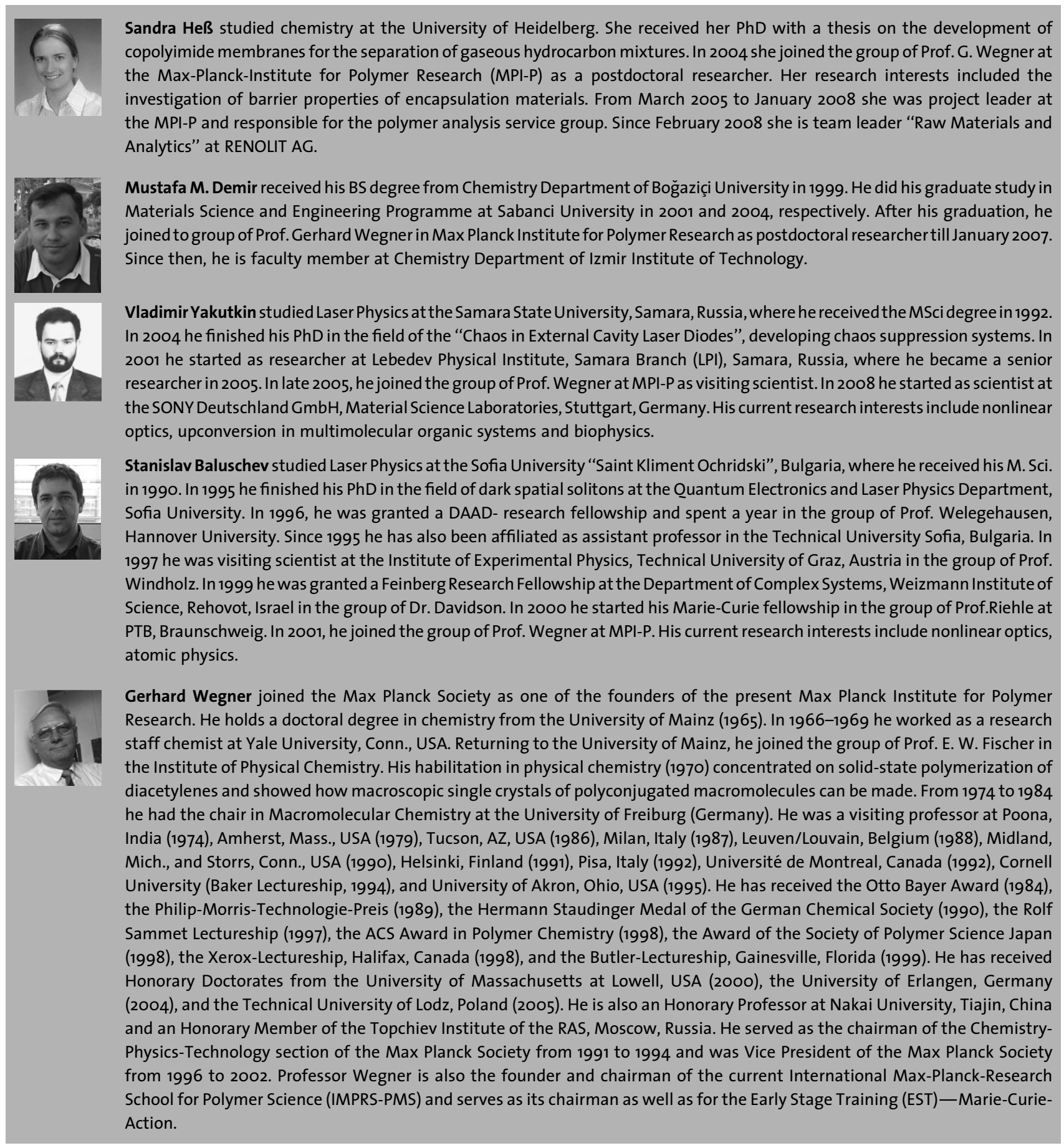

the effective cross-sectional area available for transport. Therefore, solubility of the permeating gas decreases due to loss of polymer volume available for sorption. Additionally, an increased diffusion path length occurs leading to a loss in diffusivity, i.e., the nanoparticles act as obstacles. In consequence, the enhancement of the barrier property depends on the volume fraction of nanoparticles present in the polymer matrix. A good agreement with this model was found for the propane permeability of a system consisting of natural rubber filled with $\mathrm{ZnO}$ particles. ${ }^{[3]}$

However, also the shape of the particles affects this obstacle effect. This is taken into account in Nielsons model describing the effect of plate-like particles on gas permeability in polymers. ${ }^{[4]}$ Accordingly, it is not only the volume fraction of the nanoparticles in the composite which affects the barrier properties, but also the aspect ratio of the filler plays an important role: the higher the 
aspect ratio the better the barrier properties, due to the higher surface area of the nanoparticles. The Nielson equation is presented in Equation (1), where $P_{\text {nc }}$ denotes the permeability of the nanocomposite, $P_{\mathrm{p}}$ the permeability of the pure matrix polymer, $\phi$ the volume fraction of nanofiller, and $\alpha$ the aspect ratio.

$$
P_{\mathrm{nc}}=P_{\mathrm{p}}\left(\frac{1-\phi}{1+\alpha \cdot \phi / 2}\right)
$$

We note in passing that Nielsons model assumes an orientation of the plane of the platelets normal to the flow axis of the permeating gas, i.e., the maximum barrier effect of such clay particles is assumed. ${ }^{[4,6]}$ In case of an aspect ratio $\alpha$ of 1 (i.e., the particle shape is spherical) the Nielson equation is equal to the Maxwell equation described above. In many cases the obstacle models are not applicable, because they neglect possible interactions between nanoparticle and polymer as well as nanoparticle and penetrating gas molecules.

In some cases a permeation behavior is found completely contrary to the predictions of these simple models and an increase in gas permeation was found by dispersing nanoparticles in polymers. Importantly, the presence of nanoparticles may affect the free volume of glassy polymers. ${ }^{[2,11,16]}$ The particles interfere with the polymer chain packing leading to an increased fraction of free volume, with the consequence of a higher permeability. ${ }^{[2,16]}$ It was also shown that the particle size of impermeable nanoparticles affects the gas permeability in high free volume polymers: at constant filler volume fraction, an increase in permeability with decreasing particle size was observed. ${ }^{[2,11]}$ It was also reported that strong particle surface interaction with the surrounding polymer chains can lower the mobility of the polymer near the particle surface which might lead to a reduced permeability ${ }^{[17-19]}$ and changes in the glass transition behavior. ${ }^{[20]}$

On the other hand, it was also suggested that in case of very weak interaction between particle surface and polymer segments the mobility of the chains may increase near those surfaces. ${ }^{[21]}$ This might lead to an increase in permeation in these regions. Alternatively, the polymer may form a narrow depletion zone surrounding the nanoparticles. ${ }^{[10]}$ This may lead to a shorter diffusion path. As a consequence, an increase in diffusivity and thus an increase in permeability may occur.

In conclusion, the permeation behavior of gases through nanocomposites depends on many diverse factors. Therefore, it is difficult to predict the permeation properties of nanocomposite materials based on simple model considerations.

In the present work, PMMA/ZnO nanocomposites are investigated. They are promising candidates for applica- tions-like antireflection coatings, UV-protecting films and as flame retardant materials. ${ }^{[22]}$ Additionally, an application as transparent or semitransparent barrier coating would be very interesting. Therefore, the oxygen permeabilities through such nanocomposite materials were determined to investigate the influence of spherical $\mathrm{ZnO}$ nanoparticles on the barrier properties of PMMA hybrid films.

\section{Background}

\section{Permeability}

The permeation of a penetrant through a dense polymer film is characterized by the permeability coefficient $P$, which is defined as the steady state flux $J$ of the penetrating molecules through the film normalized by film thickness and pressure difference $\Delta p=p_{1}-p_{2}$ of the gas component between feed side 1 and permeate side 2 of the film.

$$
P=\frac{J d}{\left(p_{1}-p_{2}\right)}
$$

Gas permeation through non-porous polymer films is generally described as a solution-diffusion process: the permeability coefficient is defined as the product of a diffusion coefficient $D$ and a solubility coefficient $S$ provided that $D$ is a constant, and thus independent of penetrant concentration in the film.

$$
P=D S
$$

\section{Method to Measure Oxygen Permeabilities}

To measure oxygen permeabilities, the same method of phosphorescence intensity quenching is used as described earlier. ${ }^{[23]}$ Platinum octaethylporphyrin (PtOEP) is used as sensor and is directly dissolved in the polymer film of interest. After monitoring the phosphorescence intensity of the film in the absence of oxygen, the film is exposed to a defined oxygen partial pressure and allowed to equilibrate. The oxygen molecules collide in the film with the excited dye molecules and quench their excess energy: a loss in intensity of phosphorescence can be observed. This diffusion-controlled (or dynamic) quenching of phosphorescence is given by the Stern-Volmer equation:

$$
\frac{I_{0}}{I}=\frac{\tau_{0}}{\tau}=1+k_{\mathrm{q}} \tau_{0}\left[\mathrm{O}_{2}\right]=1+k_{\mathrm{q}} \tau_{0} S p_{O_{2}}
$$

where $I$ denotes the intensity and $\tau$ the phosphorescence lifetime of the dye in the presence of oxygen while $I_{\mathrm{o}}$ and $\tau_{\mathrm{o}}$ are the corresponding values in the absence of oxygen. $k_{\mathrm{q}}$ is the bimolecular quenching rate constant and $\left[\mathrm{O}_{2}\right]$ the concentration of oxygen. By applying Henrys law a relation between the intensity and the oxygen partial 
pressure $p_{\mathrm{O}_{2}}$ as well as the solubility coefficient $S$ is obtained.

The quenching rate constant $k_{\mathrm{q}}$ is related to the diffusion controlled rate constant $k_{\text {diff }}$ for the formation of the encounter complex between oxygen and the excited dye In consequence $k_{\mathrm{q}}$ can be expressed as ${ }^{[24,25]}$

$$
k_{\mathrm{q}}=\alpha k_{\text {diff }}=\alpha 4 \pi N_{A} \sigma D
$$

Here, $\alpha$ is the probability of quenching in an encounter complex of the excited dye/quencher, $\sigma$ the effective diameter of the encounter complex, and $N_{A}$ is Avogadros number. $D$ is the sum of the diffusion coefficients of the interacting species. Since, the diffusion of oxygen in a polymer is much higher than the one of the dye, $D$ is set equal to the diffusion coefficient of oxygen. Combining Equation (5) and (4) yields:

$$
\frac{I_{0}}{I}=\frac{\tau_{0}}{\tau}=1+\left(4 \pi N_{A} \alpha \sigma \tau_{0}\right)(D S) p_{\mathrm{O}_{2}}
$$

Therefore, the desired permeability $P=D S$ can be calculated from the slope of the Stern-Volmer plot provided that $\tau_{0}, \alpha$, and $\sigma$ are known. To determine the $\tau_{0}$; values of the samples pulsed laser experiments were performed and for the product of $\alpha$ and $\sigma$ a value of $1 \mathrm{~nm}$ is assumed following the approach of previous authors. ${ }^{[26,27]}$

A recent example, in which this type of approach to measure oxygen permeability in nanocomposites was used, is found in the work of Lu et al. ${ }^{[28,29]}$ They investigated among others the effect of surface-modified silica nanoparticles on oxygen diffusion and permeation in poly (dimethylsiloxane) (PDMS). Spectroscopic investigations showed in case of PDMS that in the emission spectra the presence of silica in the film leads to a redshift of the peak position and a broadening of the peak compared to the peak of the neat PDMS film. Additionally, a significant increase in the unquenched lifetime was observed with increase in nanoparticle content in the films. Furthermore, the decay of PtOEP in PDMS-silica was non-exponentional in the absence of oxygen in contrast to the decay of the dye phosphorescence in the silica-free film. Lu et al. concluded that the environment of the PtOEP molecules is affected by the nanoparticles. Pulsed laser investigations indicated that a considerable fraction of the PtOEP molecules in PDMS is adsorbed to the silica. This adsorption leads further to the formation of dye aggregates.

\section{Experimental Part \\ Materials}

ZnO nanoparticles were prepared as described in earlier refs.[30-32] via transesterfication of Zinc acetate dihydrate with pentan-1-ol and surface-modified by conversion with tert-butylphosphonic acid $\left(t-\mathrm{BuPO}_{3} \mathrm{H}_{2}\right)$ and tetrabutylammonium acetate (TBAc) to obtain a hydrophobic $t$-Buphosphonate coating on the particles. To prepare the nanocomposite materials different ratios of the surface-modified $\mathrm{ZnO}$ nanoparticles of $22 \mathrm{~nm}$ average diameter were dispersed into MMA. In situ polymerization was carried out at $60{ }^{\circ} \mathrm{C}$ using AIBN as initiator. ${ }^{[22]}$ Nanocomposites with volume fractions of $\mathrm{ZnO}\left(\phi_{\mathrm{ZnO}}\right)$ of $1.24,2.37,4.68$, and $7.76 \%$ were prepared for this study.

Extensive characterization of the resulting composite materials can be found in earlier publications. ${ }^{[30-32]}$

\section{Sample Preparation}

To prepare dye-doped films for the permeability measurements a $10 \mathrm{wt} . \%$ solution of the nanocomposite in toluene was prepared and 0.5 wt.-\% (related to the weight of polymer) of PtOEP was added. The solutions were placed in an ultrasonic bath for at least 30 min to ensure a homogeneous distribution of the nanoparticles. The films were prepared by spincoating of $0.4 \mathrm{ml}$ of the respective solution at $1000 \mathrm{rpm}$ for $60 \mathrm{~s}$ resulting in films of thicknesses of approximately $3 \mu \mathrm{m}$ (Tencor P-10 step profiler). Carefully cleaned microscope slides of $6.5 \mathrm{~cm}^{2}$ were used as substrates. The films were stored in the dark in an evacuated desiccator for at least $24 \mathrm{~h}$. Then, the films were annealed at $110^{\circ} \mathrm{C}$ overnight. Before measurement the films were exposed to ultra-high vacuum (using a turbo molecular pump) for at least $12 \mathrm{~h}$ at room temperature.

\section{Characterization}

The same experimental setup was used for the permeability measurements which has been described in detail in an earlier publication. ${ }^{[23]}$ The dye in the samples was excited with light of a wavelength of $535 \mathrm{~nm}$ using a green Helium-Neon Laser (Lasos, $548 \mathrm{~nm}, 5 \mathrm{~mW}$ ) and the phosphorescence was detected with a photomultiplier (Products of Research, Inc.). The values for $\tau_{\text {o }}$ were determined by performing pulsed excitation experiments. For that purpose the sample, placed in vacuo, was excited by a $5 \mathrm{~ms}$ laser pulse $(<1 \mathrm{~mW})$ and the emission decay was monitored using a photomultiplier. The phosphorescence emission spectra under vacuum were determined using a $407 \mathrm{~nm}$ laser beam of $0.2 \mathrm{~mW}$. The glass transition temperatures of the materials were determined by differential scanning calorimetry (DSC) using a Mettler Toledo TG5 setup.

\section{Results and Discussion}

Poly(methyl methacrylate) is an excellent matrix for fillers due to its favorable processing conditions and its optical clarity. ${ }^{[32]}$ Zinc oxide ( $\mathrm{ZnO}$ ) is a colorless wide band gap semiconductor with an optical bandgap in the UV region, thus, it is an efficient UV absorber. Nanocomposites of these materials should be interesting for many potential applications, e.g., as UV protecting layers or antireflecting coatings. ${ }^{[22]}$ Here, the oxygen permeabilities of such nanocomposites were determined to investigate a possible application as barrier coating. Nanocomposites with 1.24, 
2.37, 4.68, and 7.76 vol.-\% ZnO particles were prepared. To illustrate the degree of particle filling in the polymer, the surface-to-surface interparticle distance (ID) of the particles in the monomer was estimated. For this calculation a spherical shape for the particles, a homogeneous dispersion as well as a uniform size distribution of the particles were assumed. Following equation was used

$$
\mathrm{ID}=d_{\mathrm{ZnO}}\left[\left(\frac{\pi}{6 \phi_{\mathrm{ZnO}}}\right)^{1 / 3}-1\right]
$$

where $d_{\mathrm{Zno}}$ denotes the diameter and $\phi_{\mathrm{Zno}}$ the volume fraction of the $\mathrm{ZnO}$ nanoparticles.

The calculated interparticle distances are 54.6, 39.7, 27.2, and $19.6 \mathrm{~nm}$ with increase in volume fraction.

Before conducting the oxygen permeability experiments two requirements have to be accomplished: first, a thermal treatment of the nanocomposite films was necessary: as shown previously, the gas permeability through a polymer film depends strongly on the history of the polymer. ${ }^{[23]}$ Therefore, all composite films were annealed at $110^{\circ} \mathrm{C}$ for $12 \mathrm{~h}$ and were then allowed to cool slowly down to room temperature before measurement to ensure the same history and, thus, same conditions for all samples.

Secondly, it was necessary to test if any interactions exist between the inorganic particles and the dye dissolved in the polymer matrix which may affect the permeability behavior. Therefore, phosphorescence emission spectra in the presence and absence of ZnO nanoparticles were measured, all in the absence of oxygen. Figure 1 shows that the shape of the peaks as well as the position of the peak maximum is independent of the amount of ZnO-nanoparticles dissolved in the polymer matrix. By exciting the sensor dye in ZnO-free as well as in ZnO-containing samples with a pulsed laser beam in the absence of

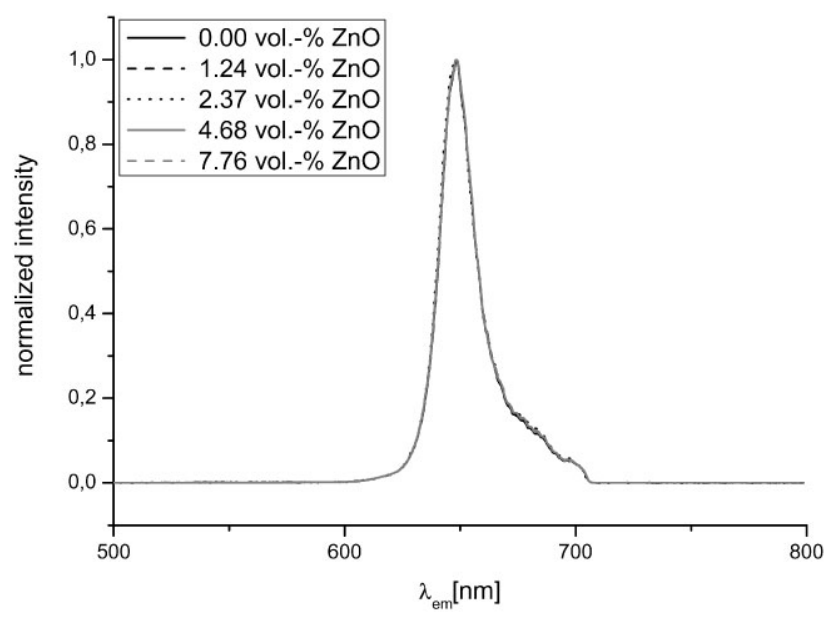

Figure 1. Phosphorescence emission spectra of PtOEP in ZnO-free and ZnO-containing PMMA samples.
Table 1. Oxygen permeabilities and glass transition temperatures of pure PMMA and its nanocomposites containing different $\mathrm{ZnO}$ contents as well as unquenched lifetimes of the excited dye dissolved in the materials.

\begin{tabular}{|c|c|c|c|}
\hline $\begin{array}{l}\text { Volume fraction of } \\
\text { ZnO in PMMA }\end{array}$ & $\tau_{\mathrm{o}}$ & $P^{\mathrm{a})}$ & $T_{\mathrm{g}}$ \\
\hline$\%$ & $\mu s$ & & ${ }^{\circ} \mathrm{C}$ \\
\hline 0.00 & 80.0 & 0.107 & 98.5 \\
\hline 1.24 & 83.3 & 0.114 & 98.0 \\
\hline 2.37 & 83.5 & 0.109 & 100.2 \\
\hline 4.68 & 80.5 & 0.114 & 97.3 \\
\hline 7.76 & 82.0 & 0.115 & 97.3 \\
\hline
\end{tabular}

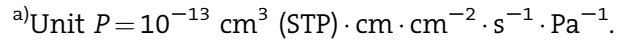

oxygen, an exponentional decay can be found in all cases. Additionally, as shown in Table 1, the unquenched lifetimes of all films are identical. These results indicate that a specific interaction of the dye with the ZnO surface does not occur, i.e., adsorption and aggregation of the dye molecule on the surface of the nanoparticles do not happen.

Gas permeation experiments based on phosphorescence quenching were conducted to determine the oxygen permeabilities of the films. The measurement starts by detecting the phosphorescence intensity of the excited sample under vacuum for at least $5 \mathrm{~min}$. Then, the desired oxygen partial pressure is applied. Now phosphorescence quenching occurs and a loss in intensity can be observed until steady state intensity is reached. A typical dataset of such decay in phosphorescence emission is shown in Figure 2. After several minutes the chamber is evacuated again to start the next measurement cycle. Usually, the measurement of one sample consists of five of such cycles by applying different gas mixtures at oxygen contents between 2.5 and $20 \%$.

The Stern-Volmer plots of all samples investigated are presented in Figure 3. The slopes of the plots of the pure PMMA film as well as of the films containing different ZnO particle contents are very similar. As stated above in Method to Measure Oxygen Permeabilities Section, the desired permeability coefficients can be calculated from the slope of these plots. Therefore, as shown in Table 1, similar values can be found for all samples. Thus, no noteworthy influence of the presence of the nanoparticles on the barrier properties of PMMA films can be observed.

Figure 4 displays TEM images of thin sections of the different nanocomposites (in the absence of PtOEP). Although, the particles were well dispersed in the 


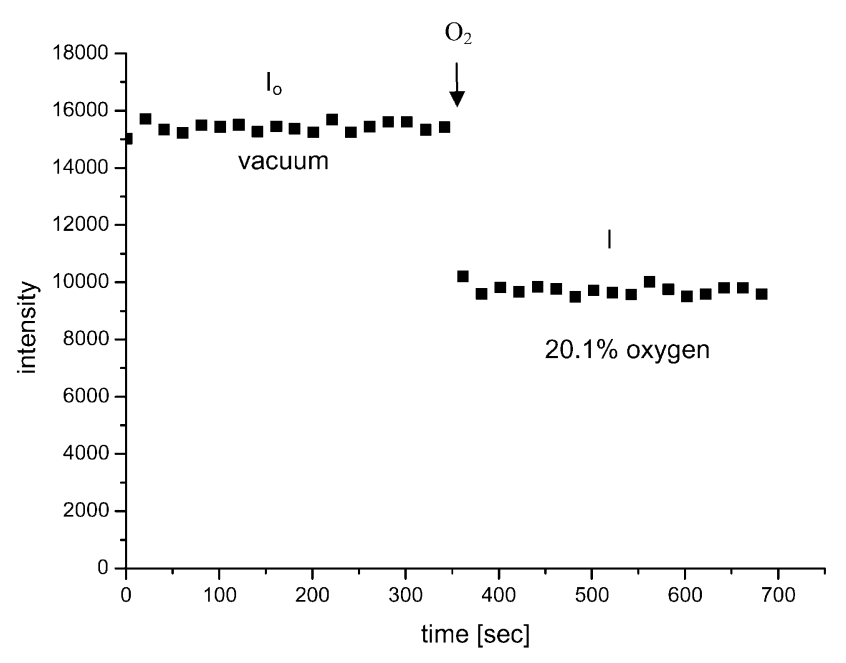

Figure 2. Decay of phosphorescence emission (PtOEP-doped $\mathrm{PMMA} / \mathrm{ZnO}$ nanocomposite film containing 4.68 vol.-\% $\mathrm{ZnO}$ ).

monomer, aggregation of the particles can be observed in the corresponding polymers. ${ }^{[22]}$ At low ZnO content (Figure $4 \mathrm{a}$ and b) separated inorganic domains of loosely associated primary particles are formed which are surrounded by large areas of ZnO-depleted PMMA. Increasing the particle volume fraction leads to a fractal type of aggregation with increasing domain sizes and an overlapping of the aggregates can be observed (Figure 4c). From the sample with volume fraction of $4.68 \%$ Figure $4 \mathrm{c}$ ) ZnO percolation of the domains can be observed. However, even the sample with the highest particle content shows a homogeneous dispersion of the aggregates and no micronsized domains are formed (Figure 4d). Nevertheless, the particle-depleted PMMA regions of this sample form

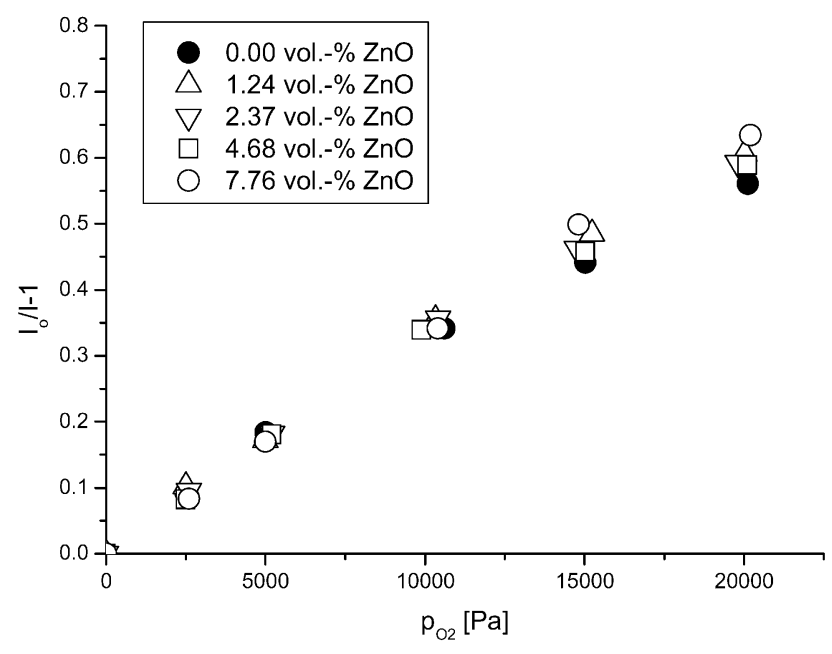

Figure 3. Stern-Volmer plots of nanocomposite samples containing different amounts of $\mathrm{ZnO}$ particles (slopes used to calculate the desired permeability coefficients, see text).
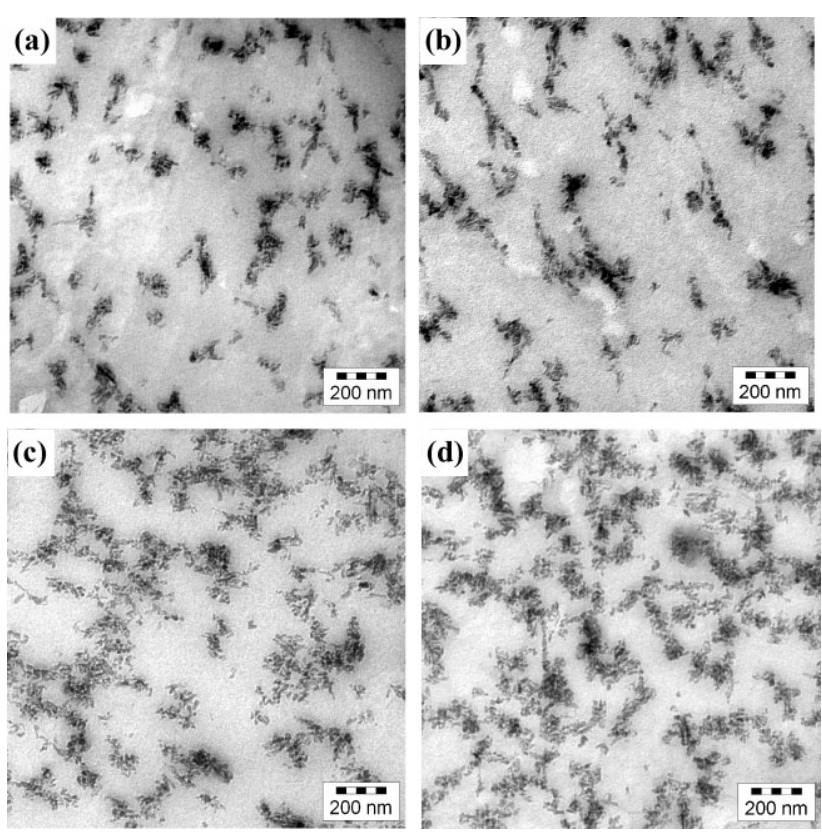

Figure 4. TEM pictures ( $b$ from Demir et al. ${ }^{[22]}$ ) of nanocomposites (without any PtOEP).

percolating domains, too, which are still clearly larger than the ZnO aggregates.

In summary, the images show regions which are enriched with nanoparticles as well as particle-depleted polymer domains. The formation of ZnO aggregates occurs as a consequence of depletion attraction. ${ }^{[31,33]}$ This phenomenon is illustrated in Figure $5 .{ }^{[31]}$ The mass center of a polymer coil is excluded from coming closer to the surface of the nanoparticle than a certain distance due to the high entropic cost of configurational distortion of the polymer chain. As a consequence, a depletion zone surrounds the nanoparticle in which no polymer center of mass can be found. If the surfaces of two nanoparticles are closer than twice the size of the depletion zone, no polymer is present in the lens-shaped region between the colloids (see Figure 5) and an osmotic force presses the

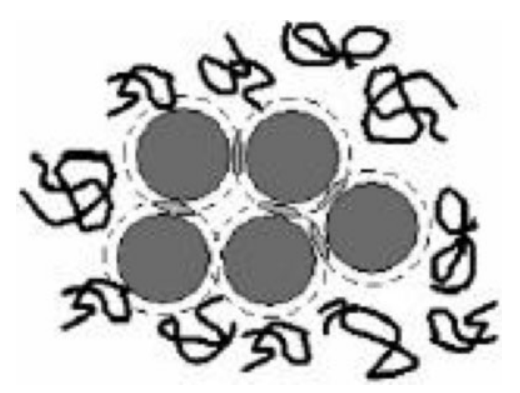

Figure 5. Cartoon presenting the depletion mechanism ${ }^{[30]}$ (see text). 
nanoparticles together-depletion attraction occurs. It seems that this depletion phenomenon leads to such a degree of aggregation that the remaining particle depleted matrix dominates the permeability to an extent that the particles are irrelevant to the flow of oxygen.

As shown above, Lu et al. suggested that the obstacle effect of nanoparticles on the permeability is compensated by another effect which increases the permeation rate: the enhanced mobility of polymer chains which surrounds the particle in case of weak interactions between colloid and polymer. In this case a decrease in glass transition temperature should be expected. Table 1 shows the glass transition temperatures $\left(T_{\mathrm{g}}\right)$ of our nanocomposites containing different amounts of nanoparticles. It can be seen that all glass transition temperatures are very similar. This result indicates that the polymer chain mobility is not affected by the presence of nanoparticles.

Oxygen molecules possibly adsorbed on the surface of ZnO aggregates cannot be detected by the sensor dye molecules which are only distributed in the polymer matrix. However, if a significant amount of oxygen molecules adsorb onto the $\mathrm{ZnO}$ surface, with increase in amount of nanoparticles a decrease in permeability should be found, because the more nanoparticles are present in the film the more oxygen should be adsorbed and the less oxygen molecules would permeate through the particle depleted zones of the composite. This effect was not observed in our case.

\section{Conclusion}

A method based on phosphorescence quenching was applied to investigate the oxygen barrier effect of PMMA/ ZnO nanocomposites with different particle contents. It was shown that the dye molecules are not adsorbed onto the ZnO particle surface but are distributed in the polymer matrix. Therefore, it can be concluded that the presence of the nanoparticles did not affect the response of the sensor dye. The experiments showed that all samples investigated have nearly the same permeability values. Additionally, no change in the glass transition temperatures was found. Therefore, it seems that neither an obstacle effect nor a significant change in polymer mobility occur affecting the permeation of oxygen through the samples. This observation can be explained by the occurrence of a depletion effect during the nanocomposite preparation leading to particle-reach and particle-depleted regions. It seems that the particle-free domains are large enough that the gas molecule can pass them without significant hindrance. The experimental result that an obstacle effect is not seen has its origin in depletion segregation of the particles. One may speculate that suppression of this effect by furbishing the particle surface with functional groups which enhance the polymer particle interaction by enthalpic forces would potentially be welcome in order to realize a barrier effect.

Received: November 25, 2008; Accepted: November 26, 2008; DOI: 10.1002/marc.200800732

Keywords: modeling; oxygen permeability; phosphorescence quenching; poly(methyl methacrylate); zinc oxide nanoparticles

[1] G. Kickelbick, Prog. Polym. Sci. 2003, 28, 83.

[2] T. C. Merkel, B. D. Freeman, R. J. Spontak, Z. He, I. Pinnau, P. Meakin, A. J. Hill, Science 2002, 296, 519.

[3] R. M. Barrer, "Diffusion in Polymers", J. Crank, G. S. Park, Eds., Academic Press, New York 1968, p. 165.

[4] J. C. Matayabas, Jr., S. R. Turner, "Polymer-Clay Nanocomposites", T. J. Pinnavia, G. W. Beall, Eds., John Wiley and Sons, Itd, Chichester 2000, p. 207.

[5] C. E. Powell, G. W. Beall, Curr. Opin. Solid State Mater. Sci. 2006, $10,73$.

[6] M. A. Osman, A. Atallah, Macromol. Rapid Commun. 2004, 25 , 1540.

[7] M. A. Osman, V. Vittal, H. R. Lusti, Macrom. Rapid Commun. 2004, 25, 1145

[8] J. Lange, Y. Wyser, Packag. Technol. Sci. 2003, 16, 149.

[9] J.-M. Yeh, S.-J. Liou, M.-C. Lai, Y.-W. Chang, C.-Y. Huang, C.-P. Chen, J.-H. Jaw, Y.-H. Yu, J. Appl. Polym. Sci. 2004, 94, 1936.

[10] H. Cong, M. Radosz, B. F. Towler, Y. Shen, Sep. Purif. Technol. 2007, 55, 281.

[11] A. L. Andrady, T. C. Merkel, L. G. Toy, Macromolecules 2004, 37, 4329.

[12] M. Moaddeb, W. Koros, J. Membr. Sci. 1997, 125, 143.

[13] J. H. Kim, Y. M. Lee, J. Membr. Sci. 2001, 193, 205.

[14] S. S. Hosseini, Y. Li, T.-S. Chung, Y. Liu, J. Membr. Sci. 2007, 302, 207.

[15] Y. Kong, H. Du, J. Yang, D. Shi, Y. Wang, Y. Zhang, W. Xin, Desalination 2002, 146, 49.

[16] P. Winberg, K. DeSitter, C. Dotremont, S. Mullens, I. F. J. Vankelecom, F. H. J. Maurer, Macromolecules 2005, 38, 3776.

[17] V. M. Litvinov, H. W. Spiess, Makromol. Chem. 1992, 193, 1181.

[18] V. M. Litvinov, H. W. Spiess, Makromol. Chem. 1991, 192, 3005.

[19] K. U. Kirst, F. Kremer, V. M. Litvinov, Macromolecules 1993, 26, 975.

[20] G. Tsagaropoulos, A. Eisenberg, Macromolecules 1995, 28, 396.

[21] D. Long, F. Lequeux, Eur. Phys. J. E 2001, 4, 371.

[22] M. M. Demir, M. Memesa, P. Castignolles, G. Wegner, Macromol. Rapid Commun. 2006, 27, 371.

[23] S. Hess, A. Becker, S. Baluschev, V. Yakutkin, G. Wegner, Macromol. Chem. Phys. 2007, 208, 2173.

[24] M. Smoluchowski, Zeitschr. f. physik. Chemie 1917, 92, 129.

[25] J. E. Guillet, "Photophysical And Photochemical Tools in Polymer Science", M. A. Winnik, Ed., Kluwer, Dordrecht 1986, p. 467.

[26] Z. Masoumi, V. Stoeva, A. Yekta, Z. Pang, I. Manners, M. A. Winnik, Chem. Phys. Lett. 1996, 261, 551.

[27] B.-H. Han, M. A. Winnik, A. B. Bourlinos, E. P. Giannelis, Chem. Mater. 2005, 17, 4001. 
[28] X. Lu, I. Manners, M. A. Winnik, Macromolecules 2001, 34 , 1917.

[29] X. Lu, M. A. Winnik, Chem. Mater. 2001, 13, 3449.

[30] M. M. Demir, R. Munoz-Espi, I. Lieberwirth, G. Wegner, J. Mater. Chem. 2006, 16, 2940.
[31] M. M. Demir, P. Castignolles, Ü. Akbey, G. Wegner, Macromolecules 2007, 40, 4190.

[32] M. M. Demir, K. Koynov, Ü. Akbey, C. Bubeck, I. Park, I. Lieberwirth, G. Wegner, Macromolecules 2007, 40, 1089.

[33] W. C. K. Poon, J. Phys.: Condens. Matter. 2002, 14, R859. 